\title{
The correlation between the central carbon metabolic flux distribution and the number of shared enzyme regulators in Saccharomyces cerevisiae
}

\author{
Xiangfei Zhou ${ }^{1}$, Lunxian Liu ${ }^{1}$, Chuanyu Shang ${ }^{1}$, Haifeng $\mathrm{Xu}^{1}$, Chao Ding ${ }^{1}$, Qian Liu ${ }^{1}$, Yin \\ $\mathbf{Y i}^{\mathbf{1}^{*}}$ \\ ${ }^{I}$ School of Sciences, Guizhou NormalUniversity, Guiyang, China, 2 Key Laboratory of Plant Physiology and \\ Developmental Regulation of Guizhou Province, Guiyang, China.
}

\begin{abstract}
The central carbon metabolic system is the upstream energy source for microbial fermentation. In addition, it is a master switch for increasing the production of metabolites and an important part of the microbial metabolic network. Investigation into the relationship between genes, environmental factors, and metabolic networks is a main focus of systems biology, which significantly impacts research in biochemistry, metabolic engineering, and synthetic biology. To this end, the central carbon metabolic flux under a variety of growth conditions or using strains with various genetic modifications was previously measured in Saccharomyces cerevisiae using ${ }^{13} \mathrm{C}$ tracer technology. However, the measured values were not integrated and investigated further. In this study, we collected and analyzed the metabolic flux rates of the central carbon metabolic system in $S$. cerevisiae measured in recent studies. We carried out preliminary analyses of flux values of each pathway, performed regression analyses on relationship between different fluxes, and extracted principal component factors of the flux variables. Based on the results, the general characteristics of pathway flux distribution were clustered and explored, and the effects of environmental and genetic factors on the flux distribution were analyzed. Furthermore, this study explored the relationship between similarity in the enzyme's transcriptional regulation and the correlations in the enzyme's reaction flux. Our results provide a foundation for further studies on the control of the central carbon metabolic flux and facilitate the search for targets in metabolic engineering research.
\end{abstract}

Key words: Saccharomyces cerevisiae, central carbon metabolic flux, metabolic networks and pathways, gene expression regulation, gene environment interaction, enzyme transcriptional regulator

${ }^{1}$ Authors for correspondence: gzklppdr@gznu.edu.cn 


\section{INTRODUCTION}

Saccharomyces cerevisiae is a single-celled fungal microorganism that has been used by humans from the earliest times. Because it reproduces asexually by budding, it is also known as budding yeast. $S$. cerevisiae is an important biotechnological organism for the production of ethanol, proteins, antibiotics, fatty acids, or other biochemical substances (Kato et al. 1998; Nissen et al. 2000; Koh et al. 2002; Kim et al. 2004; Leber and Da Silva 2014). S. cerevisiae has decisive advantages in industrial processes due to its tolerance to alcohols and fermentation conditions (Generoso et al. 2015). These characteristics have made $S$. cerevisiae appealing for production of industrially important biochemicals (Nevoigt 2008). In addition, as a simple eukaryote, $S$. cerevisiae is often used as a model organism for research on eukaryotic metabolism (Dujon 1998; Oliver 2002), which has led to high interest in understanding metabolic function and regulation in this organism. Over the past few decades, researchers have gradually increased the yield and/or production rate of valuable target metabolites by transforming genes into $S$. cerevisiae using genetic engineering techniques and improving the methods of $S$. cerevisiae fermentation (Chiang et al. 2013). These strategies have often been based upon overcoming the natural feedback regulation mechanisms specific to each desired biosynthetic pathway sequence. It is now apparent that further improvements will necessitate a more complete knowledge of the factors influencing carbon flux through the central pathways so as to more efficiently supply the specific biosynthetic pathways with the necessary carbon precursors and coenzymes.

Classic central carbon metabolism includes pathways such as the adenosine monophosphate pathway, the pentose phosphate pathway, and the tricarboxylic acid cycle (Sudarsan et al. 2014) (Fig 1). These metabolic pathways are the main sources of energy required by the organism, and they provide precursors for other metabolic pathways (Bennett et al. 2009). Therefore, the central carbon metabolism system is responsible for microbial fermentation; it is also an essential step and the primary switch for improving the production of metabolites (Shi et al. 2014; Zhang et al. 2014).

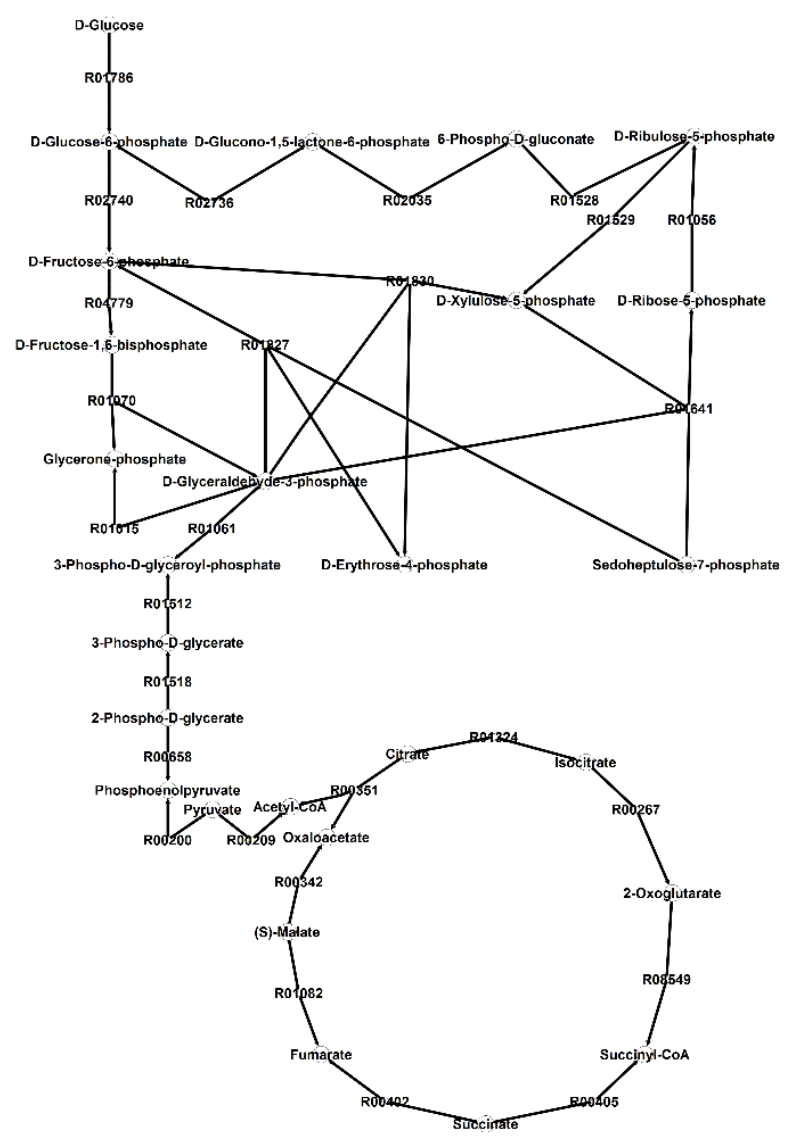

Figure 1 - The reaction network for the central carbon metabolism.

Investigation of the metabolic regulatory properties of $S$. cerevisiae has made a critical contribution toward optimization of strains and production processes.

Given the importance of the central carbon metabolism in controlling the total production of metabolites and their production efficiency, an accurate quantification of the central carbon metabolic flux of $S$. cerevisiae under various conditions would help us to understand its regulatory mechanism. Fluxes of biochemical reactions and pathways can be determined most extensively and precisely using ${ }^{13} \mathrm{C}$ isotopic labeling techniques. To obtain detailed information on fluxes through central metabolism, ${ }^{13} \mathrm{C}$ isotopomer analysis is generally combined with a stoichiometric reaction network. Such approaches have proven useful for the elucidation of the global functional and regulatory activities of cells (Stephanopoulos et al. 1998). In recent years, 
researchers have measured the central carbon metabolic flux status using the ${ }^{13} \mathrm{C}$ tracer technology under various nutritional environments and/or using $S$. cerevisiae strains with genetic modifications, e.g., "Comparative genomic analysis of Saccharomyces cerevisiae yeasts isolated from fermentations of traditional beverages unveils different adaptive strategies." However, there has been no integrated analysis or comparison of these measured flux values to our knowledge.

Therefore, in this study, we collected the central carbon metabolism flux values of the $S$. cerevisiae system from recent studies, carried out preliminary analysis of the distribution of flux values in each pathway, studied the regression relationships among different fluxes, extracted the main component factors of the flux vector, summed the general characteristics of the central carbon metabolism pathway flux distribution, and investigated the impact of environmental and genetic factors over flux distributions. In particular, a significant quantitative relationship was found between the fluxes and the number of shared regulators of the enzymes that catalyzed the flux. This interesting finding provides new material for further research on the characteristics of central carbon metabolism flux distribution and control.

\section{MATERIALS AND METHODS}

\section{Data source}

The PubMed reference database was queried using various combinations of keywords such as " ${ }^{13} \mathrm{C}$," "metabolic," "flux," "Saccharomyces cerevisiae," "central carbon," and "analysis," returning approximately 140 literature results from 1991 to 2014. Sixty-five percent of the studies were automatically excluded due to the absence of quantitative flux distribution information, and a further $20 \%$ were excluded for measurements using a non- ${ }^{13} \mathrm{C}$ method. Ultimately, a total of 14 references were collected as a preliminary source for our database (Gombert et al. 2001; Pitkänen et al. 2003; dos Santos et al. 2003; Cakir et al. 2004; Daran-Lapujade et al. 2004; Sondereqqer et al. 2004; Raghevendran et al. 2004; Grotkjær et al. 2005; Blank et al. 2005; Kuepfer et al. 2005; Frick and Wittmann 2005; Fendt and Sauer 2010; Papini et al. 2012; Nagamori et al. 2013).

\section{Network definition}

The bioreaction network was adopted from previous work. The metabolic pathway includes the Embden-Meyerhof pathway, pentose phosphate pathway, tricarboxylic acid cycle, anaplerotic reaction, and glyoxylate shunt.

\section{Flux value formatting and assembly}

The diversity of the reactions and network definition, the quantity of experimental data, and the required genetic and cultivation knowledge made the assembly of the CeCaFDB both difficult and time consuming(Zhang et al. 2014). The lumped reactions in these studies were broken down into their original forms, as found in the Kyoto Encyclopedia of Genes and Genomes (KEGG) reaction database, and the flux value was mapped to its precisely corresponding reaction. About 64 groups of flux values from 14 studies were acquired (Table 1).

Table 1 - The literature source of metabolic flux data in this paper.

\section{Number}

The name of each Article title
A1
Characterization of the metabolic shift between oxidative and fermentative growth in
Saccharomyces cerevisiae by comparative $13^{\mathrm{C}}$ flux analysis (Frick and Wittmann 2005)
Comparative metabolic network analysis of two xylose fermenting recombinant Saccharomyces cerevisiae strains (Grotkjær et al. 2005) Saccharomyces cerevisiae by Using 13 ${ }^{\mathrm{C}}$-Labeled Substrates (dos Santos et al. 2003) 
A5

A6

A7

A8

A10

A11

A12

A13

A14

Metabolic flux analysis of genetically engineered Saccharomyces cerevisiae that produces lactate under micro-aerobic Conditions(Nagamori et al. 2013)

Metabolic flux analysis of xylose metabolism in recombinant Saccharomyces cerevisiae using Continuous culture(Pitkänen et al. 2003)

Metabolic functions of duplicate genes in Saccharomyces cerevisiae(Kuepfer et al. 2005)

Metabolic Pathway Analysis of Yeast Strengthens the Bridge Between Transcriptomics and Metabolic Networks(Cakir et al. 2004)

Molecular Basis for Anaerobic Growth of Saccharomyces cerevisiae on Xylose,Investigated by Global Gene Expression and Metabolic Flux Analysis(Sondereqqer et al. 2004)

Network Identification and Flux Quantification in the Central Metabolism of Saccharomyces cerevisiae under Different Condition s of Glucose Repression(Gombert et al. 2001)

Phenotypic characterization of glucose repression mutants of Saccharomyces cerevisiae using experiments with $13^{\mathrm{C}}$-labelled glucose(Raghevendran et al. 2004)

Physiological characterization of recombinant Saccharomyces cerevisiae expressing the Aspergillus nidulans phosphoketolase pathway :validation of activity through $13^{\mathrm{C}}$-based metabolic flux analysis(Papini et al. 2012)

Role of Transcriptional Regulation in Controlling Fluxes in Central Carbon Metabolism of Saccharomyces cerevisiae:A CHEMOSTAT CULTURE STUDY(Daran-Lapujade et al. 2004)

Transcriptional regulation of respiration in yeast metabolizing differently repressive carbon substrates(Fendt and Sauer 2010)

\section{Multiple-regression padding flux data}

Since specific culture conditions and experimental objectives varied among experiments, there were some discrepancies in the metabolic pathway flux values from published data. Therefore, we considered how to match the data for statistical analysis in each sample. The strategy we took was to build a multiple linear regression equation where the unknown flux was taken as the unknown variable and the known flux as the predictive variable according to the samples. Thus, each unknown flux was determined in each metabolic flux case. SPSS 19.0 was used as the regression tool, and its exit method (stepwise method) was used to build the regression equation.

\section{Principal component analysis}

The metabolic flux values of all pathways in each case were set as objects using the PRINCOMP procedure in MATLAB to perform principal component analysis and obtain eigenvectors and eigenvalues (Nakayama et al. 2014). The resultant principal components were sorted according to their eigenvalues, and a scree map was constructed.
Based on this map, a component conversion matrix was built in which the three largest principal components (PC1, PC2, and PC3) were selected to form a scatter diagram showing the distribution of all samples in the space of main components (PC1, PC2, PC3).

\section{Regulator inquiry}

Each enzyme's transcriptional regulators, identified from microarray data, were searched for in the Saccharomyces genome database (SGD). The relationship between transcriptional regulation of enzymes involved in central carbon metabolism and the changes in their enzymatic reaction fluxes as well as the relationship between enzyme amounts and their reaction fluxes were analyzed.

\section{RESULT}

\section{Data padding}

In previous studies, experimental data were measured in different strains of $S$. cerevisiae under different conditions based on different experimental objectives. This creates a problem for analysis of 
combined studies because some data in the literature mainly measure the flux of a portion of the bypass of the central carbon metabolism system, whereas others measure the flux of a different portion. This leads to a situation where the number of bypasses tested in different studies was relatively small, thereby limiting the comparisons of the flux values.

To compare flux values, the unmeasured flux data can be derived via the regression method by using the flux data that are already available in the literature. For example, in study X (Physiological characterization of recombinant $S$. cerevisiae expressing the Aspergillus nidulans phosphoketolase pathway: validation of activity through ${ }^{13} \mathrm{C}$-based metabolic flux analysis), the flux values of reactions R01529 (KEGG reaction code) and R01056, which control the conversion of Dribulose-5-phosphate to D-ribose-5-phosphate, were not measured, but all other flux values were determined; additionally, the flux values of R01529, R01056, and another 15 reactions were determined in other studies (Papini et al. 2012). Thus, using these flux values and the exit regression method, the regression equations of the two reactions could be established. The values of R01529 and R01056 could then be inferred based on the flux values in document $\mathrm{X}$.

Similarly, based on the different combinations of known and unknown fluxes from different studies, the regression equations for R02740, R01518, R00658，R00209，R00351，R01324，R00267, R08549, R00405, and R00402 were established. Although these derived values are not completely accurate, they play an important role in the subsequent analysis.

\section{The flux value distribution}

Based on the padded data set, the basic statistical analysis of the flux values for each reaction was carried out, and the variability and the average of the fluxes were compared.

The magnitude of the flux value of each reaction is indicated by the standard deviation of the flow value, which can be divided into three major types (Fig. 2). The change in magnitude of the flux value for the first type of reaction was relatively large, and its standard deviation varied from 220 to 520 . Except for R01082, all other reactions of this type were mainly concentrated in the glycolysis and pentose phosphate pathways. Since the pentose phosphate bypass consumes glucose-6-phosphate and complements the glycolytic pathway, there exists redundancy in this relationship. Meanwhile, since the main reaction was reversible, a large variation in the range of flux values was inevitable. The change in flux values for the second type of reaction varied from 100 to 200 , and these were involved primarily in the tricarboxylic acid cycle. Because of the lower overall carbon flux of the tricarboxylic acid cycle, its range of flux changes was relatively smaller. The variation of flux for the third type of reaction was much smaller $(<100)$. These reactions were primarily involved in the nonoxidative phase of the pentose phosphate pathway. According to the SGD, there were fewer reactionassociated regulators, which might have resulted in the small variations to changes in the flux values.

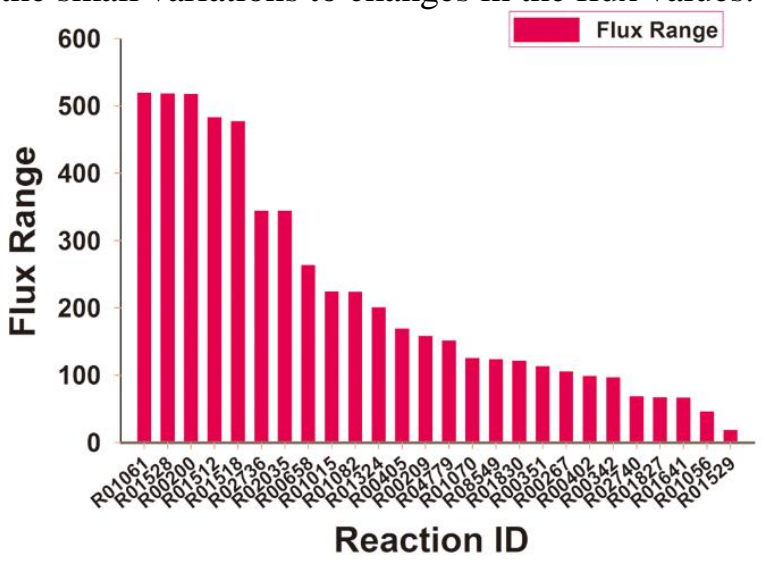

Figure 2 - Flux range of the reactions in central carbon metabolism. The reaction ID on $\mathrm{x}$ axis represents the reaction index number for different reactions. The bar value on $y$ axis is the maximal flux values minus the minimal flux values for the corresponding reaction across all data.

\section{Principal component distribution}

Principal component analysis was done on the complete data set after reconstruction. The feature value distribution of principal components is shown in Figure 3. The first feature value of the main component was 11.3 , the second was 5.7 , and the third was 3.1. The contribution of the first three principal components to the variance was $77 \%$. 




Figure 3 - Scree plot of the principle component sorted by Eigen value. The y axis represents the Eigen value for the principle components. The principle components are ordered, and by definition are therefore assigned a number label, which is the number in $\mathrm{x}$ axis.

All samples were divided into two groups based on environmental conditions or genetic changes. The experiments in the first group were conducted on wild-type strains under various growth environments. This group was called the environmental change group. The second group comprised genetically modified strains and was called the genetically modified group. The first three principal components were chosen as the coordinates, and the collection of all samples is described in Figure 4. In this figure, green dots indicate the environmental change group, and red dots indicate the genetically modified group.

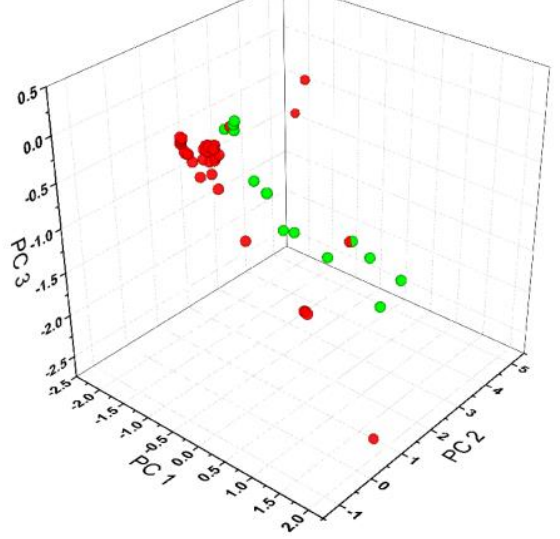

Figure 4 - Scatter plot of the flux distributions in principle component space. The $\mathrm{x}, \mathrm{y}$ and $\mathrm{z}$ axis represents the value of principle component 1,2 and 3, respectively. Red point represents the flux distribution of genetically modified strains while green point denotes the flux distribution of strains from changed environments.

\section{Relationship between enzyme transcriptional regulators and enzyme reaction flux}

Sixty-four groups of metabolic flux values were collected; the missing parts were predicted and supplemented, making this a relatively complete data set. Next, the Pearson correlation coefficients between the flux values of each reaction were calculated; the results showed that the correlation coefficients among different reactions were not completely similar. Some were relatively high, which might be of biological significance. The correlation between the fluxes, apart from the stoichiometric feature constraints of the metabolic network, might be generated from common biological constraints. The amount of enzyme is the most important biological characteristic affecting enzyme reaction flux. The transcriptional regulation of enzymes plays a decisive role among factors that can affect the number of enzyme molecules. Therefore, it naturally follows that coexistent transcriptional regulation between two enzymes affects the correlation between their fluxes.

Through searches in the SGD, the transcriptional regulator of each enzyme was identified based on microarray data. The flux correlations and the amounts of common enzyme regulators for pairwise reactions with more than four regulators were compared and constructed. The results are shown in Figure 5. In this figure, the $y$-coordinate represents the correlation coefficient, the $x$ coordinate is the regulator shared by the two enzymes, and the dot represents the pairwise reaction in a group.

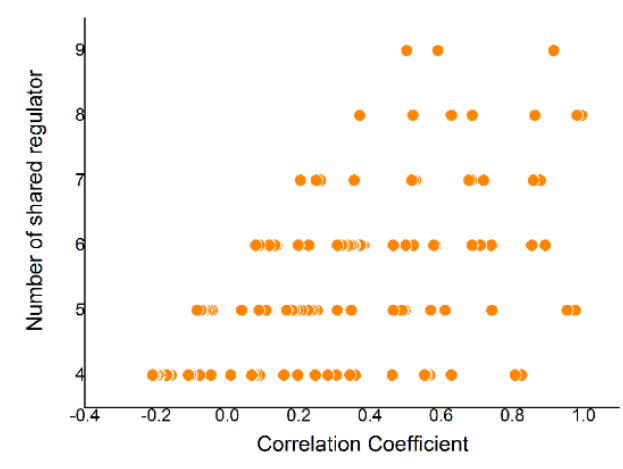

Figure 5 - The relationship between Pearson correlation coefficient between fluxes and number of shared regulator among genes. The $y$-coordinate represents the correlation coefficient, the $\mathrm{x}$-coordinate is the regulator shared by the two enzymes, and the dot represents the pairwise reaction in a group. 


\section{DISCUSSION}

The analysis of the average values of flux for each reaction resulted in a very interesting finding: the mean flux of each reaction was not entirely random but clustered around several relatively fixed values (Fig. 6). For instance, the average values of reactions involved in the glycolytic pathway were relatively large and concentrated around 80-160. This was because most of the carbon atoms did not sediment into the biomass at this time but existed in the form of three-carbon molecules; therefore, the carbon-carrying fluxes were relatively large. The value of the non-oxidative phase of the pentose phosphate pathway was very small at around 4 . Finally, the average values of the tricarboxylic acid cycle and the oxidative phase of the pentose phosphate pathway were concentrated primarily in the range of 10-30.

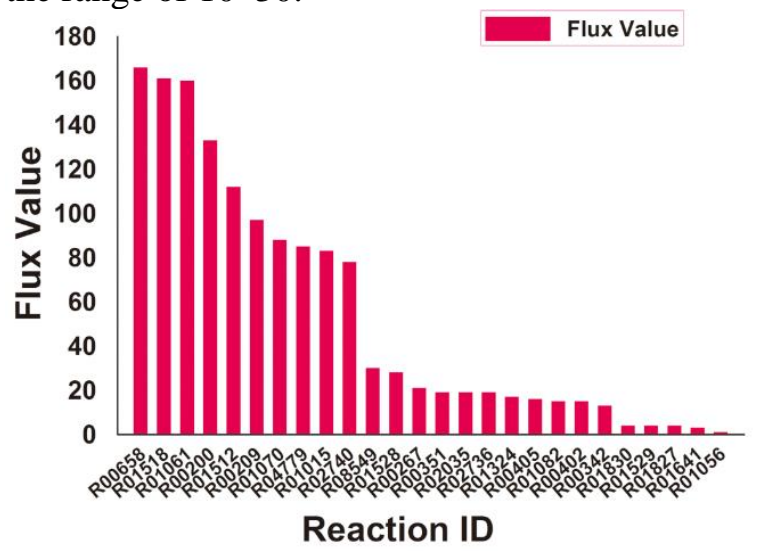

Figure 6.Average flux value of the reactions in central carbon metabolism.The reaction ID on $\mathrm{x}$ axis represents the reaction index number for different reactions. The bar value on $y$ axis is the average flux values for the corresponding reaction across all data.

This analysis identified the magnitude of reaction flux values of each enzyme under physiological conditions. For enzymes with small reaction fluxes, the total available enzyme might limit the flux. Therefore, enzyme activity must be taken into account to change the flux through this enzyme. Similar analyses can also provide other insights.

From Figure 4, the genetically and environmentally modified cases were found to be interspersed, with the distribution of fluxes in the environmentally modified group sparser than that from the genetically modified group, which points to a more stringent genetic effect on the flux distribution compared with that of environmental changes. In addition, we found that genetic modifications had a stronger impact on certain flow values. The PC1 values of multiple genetically modified strains were below -2 , whereas this situation rarely occurred in samples from the environmental change group.

As shown in Figure 5, the number of shared regulators affects the correlation coefficient on two levels. First, it determines the range of distributions of the correlation coefficient. Larger numbers of shared regulators correlated to increased biological association, a larger minimum value of the correlation coefficient, and a smaller range of the correlation coefficient distribution. The inverse was also true: smaller numbers of shared regulators correlated to a lower degree of biological association, a smaller minimum value of the correlation coefficient, and a larger distribution range of correlation coefficients. There was an almost linear relationship between the number of shared regulators and the minimum value of the correlation coefficient. This also determines the average value of correlation coefficients: a positive correlation was observed between the number of shared regulators and the average value of the correlation coefficient. The results indicate an obvious association between an enzyme's transcriptional regulation and changes in its reaction flux. The results also indicate that there is a strong correlation between the number of enzymes and the enzymatic reaction flux. These insights can facilitate the search for targets in metabolic engineering research.

\section{ACKNOWLEDGMENTS}

This work was supported by the Key Laboratory construction project of science and Technology Department of Guizhou Province (Grant NO. 20114005); Social development project of science and Technology Department of Guizhou Province (Grant NO. 2011-3068); the 125 major scientific and technological projects of Guizhou Provincial education department (Grant NO. 2012-005); Key Basic Research Project of Guizhou Province, China (Grant NO. 2014-2001); Social development project of science and Technology Department of Guizhou Province (Grant NO. 2012-3180).

\section{AUTHOR CONTRIBUTIONS}

This work was supported by the Key Laboratory construction project of science and Technology Department of Guizhou Province (Grant NO. 2011?4005); Social development project of science 
and Technology Department of Guizhou Province (Grant NO. 2011?3068); the 125 major scientific and technological projects of Guizhou Provincial education department (Grant NO. 2012?005); Key Basic Research Project of Guizhou Province, China (Grant NO. 2014?2001); Social development project of science, Technology Department of Guizhou Province (Grant NO. 2012?3180) and the Joint Fund of Guizhou Bureau of Science and Technology (Qiankehe LH[2015] 7773).

\section{REFERENCES}

Bennett BD, Kimball EH, Gao M, Osterhout R, Van Dien SJ,Rabinowitz JD. Absolute Metabolite Concentrations and Implied Enzyme Active Site Occupancy in Escherichia coli. Nat. Chem. Biol. 2009;5(8):593-599.

Blank LM,Kuepfer L,Sauer U. Large-scale ${ }^{13}$ C-flux analysis reveals mechanistic principles of metabolic network robustness to null mutations in yeast. Genome Biology. 2005;6(6):49.

Cakir T, Kirdar B, Ulgen KO. Metabolic Pathway Analysis of Yeast Strengthens the Bridge Between Transcriptomics and Metabolic Networks. Biotechnol Bioeng. 2004;89(3):251-260.

Chiang CJ,Yeh GL,Chen PT,LinTH,Hwang WS,Chao YP. Development of a genomic engineering tool in Saccharomyces cerevisiae. Journal of the Taiwan Institute of Chemical Engineers.2013;45(1):24-31.

Daran-Lapujade P,Jansen ML,Daran JM,van Gulik W,de Winde JH,Pronk JT. Role of Transcriptional Regulation in Controlling Fluxes in Central Carbon Metabolism of Saccharomyces cerevisiae:A CHEMOSTAT CULTURE STUDY. J. Biol.Chem. 2004;279(10):9125-9238.

dos Santos MM, Gombert AK, Christensen B,Olsson L, Nielsen J .Identification of In Vivo Enzyme Activities in the Cometabolism of Glucose and Acetate by Saccharomyces cerevisiae by Using ${ }^{13} \mathrm{C}$-Labeled Substrates. Eukaryotic Cell. 2003;2(3):599-608.

Dujon B.European Functional Analysis Network (EUROFAN) and the functional analysis of the Saccharomyces cerevisiae genome. Electrophoresis.1998; 19:617-624.

Fendt SM, Sauer U.Transcriptional regulation of respiration in yeast metabolizing differently repressive carbon substrates. BMC Systems Biology.2010;4(1):111.

Frick O,Wittmann C.Characterization of the metabolic shift between oxidative and fermentative growth in Saccharomyces cerevisiae by comparative $13^{\mathrm{C}}$ flux analysis. Microbial Cell Factories. 2005; 4:30.

Generoso WC,Schadeweg V, Oreb M, Boles E. Metabolic engineering of Saccharomyces cerevisiae for production of butanol isomers. Curr Opin Biotechnol. 2015; 33:1-7.
Gombert AK,Moreira dos Santos M,Christensen B,Nielsen J. Network Identification and Flux Quantification in the Central Metabolism of Saccharomyces cerevisiae under Different Condition s of Glucose Repression. J. Bacteriol. 2001;183(4):1441-1451.

Grotkjær T,Christakopoulos P,Nielsen J, Olsson L.Comparative metabolic network analysis of two xylose fermenting Recombinant Saccharomyces cerevisiae strains. Metab Eng .2005;7(5-6):437-444.

Kato A, Nakamura S, Ibrahim H, Matsumi T, Tsumiyama C, Kato M.Production of genetically modified lysozymes having extreme heat stability and antimicrobial activity against gram negative bacteria in yeast and in plant. Nahrung.1998;42(3-4):128-130.

Kim MD, Lee TH, Lim HK, Seo JH. Production of antithrombotic hirudin in GAL1-disrupted Saccharomyces cerevisiae. Appl Microbiol Biotechnol.2004; 65(3):259-262.

Koh JH, Yu KW, Suh HJ. Biological activities of Saccharomyces cerevisiae and fermented rice bran as feed additives. Lett Appl Microbiol.2002; 35(1):47-51.

Kuepfer L,Sauer U,Blank LM.Metabolic functions of duplicate genes in Saccharomyces cerevisiae. Genome Res.2005;15(10): 1421-1430.

Leber C, Da Silva NA. Engineering of Saccharomyces cerevisiae for the Synthesis of Short Chain Fatty Acids. Biotechnol Bioeng. 2014;111(2):347-358.

Nagamori E, Shimizu K, Fujita H, Tokuhiro K, Ishida $\mathrm{N}$, Takahashi H. Metabolic flux analysis of genetically engineered Saccharomyces Cerevisiae that produces lactate under micro-aerobic conditions. Bioprocess Biosyst Eng. 2013; 36(9):1261-1265.

Nakayama Y, Putri SP, Bamba T,Fukusaki E. Metabolic distance estimation based on principle component analysis of metabolic tumover. J Biosci Bioeng. 2014(3),118: 350-5.

Nevoigt E. Progress in metabolic engineering of Saccharomyces cerevisiae. Microbiol Mol Biol Rev 2008;(3),72:379.

Nissen TL, Kielland-Brandt MC, Nielsen J, Villadsen J. Optimization of ethanol production in Saccharomyces cerevisiae by metabolic engineering of the ammonium assimilation.Metab Eng .2000;2(1):69-77.

Oliver SG. Functional genomics: lessons from yeast. Philos Trans $R$ Soc Lond B Biol Sci. 2002;357(1417):17-23.

Papini M, Nookaew I, Siewers , Nielsen J.Physiological characterization of recombinant Saccharomyces cerevisiae expressing the Aspergillus nidulans phosphoketolase pathway :validation of activity through 13C-based metabolic flux analysis.Appl Microbiol Biotechnol.2012; 95:1001-1010.

Pitkänen JP, Aristidou A, Salusjärvi L, Ruohonen L.Metabolic flux analysis of xylose metabolism in recombinant Saccharomyces cerevisiae using continuous culture. Metab Eng .2003;5(1):16-31.

Raghevendran V, Gombert AK, Christensen B, Kotter 
$\mathrm{P}$,Nielsen $\mathrm{J}$.Phenotypic characterization of glucose repression mutants of Saccharomyces cerevisiae using experiments with 13C-labelled glucose. Yeast. 2004;21: 769-779.

Shi XC, Chen Y, Ren HF, Liu D, Zhao T, Zhao N et al. Economically enhanced succinic acid fermentation from cassava bagasse hydrolysate using Corynebacterium glutamicum immobilized in porous polyurethane filler. Bioresour Technol. 2014;174: 190-197.

Sondereqqer M,Jeppsson M, Hahn-Hägerdal B, Sauer U. Molecular Basis for Anaerobic Growth of Saccharomyces cerevisiae on Xylose,Investigated by Global Gene Expression and Metabolic Flux Analysis. Appl Environ Microbiol.2004;70(4):2307-2317.

Stephanopoulos GN, Aristidou AA,Nielsen J. Metabolic Engineering: Principles and Methodologies. San Diego,Academic Press, 1998.

Sudarsan S, Dethlefsen S, Blank LM, SiemannHerzberg M, Schmid A.The functional structure of central carbon metabolism in Pseudomonas putida KT2440. Appl Environ Microbiol.2014;80(17):52925303.
Zhang ZD, Shen T, Rui B, Zhou WW, Zhou XF, Shang $\mathrm{CY}$ et al. CeCaFDB: a curated database for the documentation,visualization and comparative analysis of central carbon metabolic flux distributions explored by $13^{\mathrm{C}}$-fluxomics. Nucleic Acids Res.2014;43:549557.
Received: October 19, 2015; Accepted: April 05, 2016. 